\title{
Hypoxia upregulates Hsp90 $\alpha$ expression via STAT5b in cancer cells
}

\author{
SE HYUNG PAK ${ }^{1 *}$, YOUN HEE JOUNG ${ }^{1 *}$, JIN HEE PARK ${ }^{1}$, EUN JOUNG LIM ${ }^{1}$, PRAMOD DARVIN ${ }^{1}$, \\ YOON MI NA ${ }^{1}$, DAE YOUNG HONG ${ }^{2}$, BORAM LEE $^{3}$, TAE SOOK HWANG ${ }^{1}$, TAEGYU PARK $^{4}$, \\ SANG-KYU YE ${ }^{5}$, EON-SOO MOON ${ }^{6},{\text { BYUNG WOOK } \mathrm{CHO}^{7}, \mathrm{KYUNG} \text { DO PARK }}^{8}$, \\ HAK KYO LEE ${ }^{8}$, ILL-MIN CHUNG ${ }^{9}$ and YOUNG MOK YANG ${ }^{1}$
}

\author{
Departments of ${ }^{1}$ Pathology, School of Medicine, and Institute of Biomedical Science and Technology and \\ ${ }^{2}$ Emergency Medicine, Konkuk University Hospital; ${ }^{3}$ Konkuk University Hospital, Seoul 143-701; \\ ${ }^{4}$ Bio-Food and Drug Research Center, Department of Biotechnology, College of Biomedical and Health Science, \\ Konkuk University, Chungju 380-701; ${ }^{5}$ Department of Pharmacology, School of Medicine, \\ Seoul National University, Seoul 110-799; ${ }^{6}$ Department of Internal Medicine, School of Medicine, \\ Konkuk University Glocal Campus, Chungju 380-701; ${ }^{7}$ Department of Animal Science, College of Life Sciences, \\ Pusan National University, Busan $609-735 ;{ }^{8}$ Genomic Informatics Center, Hankyong National University, Anseong 456-749; \\ ${ }^{9}$ Department of Applied Life Science, Konkuk University, Seoul 143-701, Republic of Korea
}

Received January 18, 2012; Accepted March 12, 2012

DOI: $10.3892 / \mathrm{ijo} .2012 .1450$

\begin{abstract}
Hsp90 $\alpha$ is a molecular chaperone protein involved in the structural maturation of oncogenic signaling proteins. Hsp90 was recently identified as an anticancer target; various studies are ongoing to find ways for managing cancer through Hsp $90 \alpha$. However, this approach is limited by reported sideeffects. Hypoxia is a hallmark of solid tumors, including those of breast cancer and the extent of tumor hypoxia is associated with resistance to treatment and poor prognosis. One of the major signaling pathways in cancer cells, the Jak2/STAT5b pathway, has been found to be closely correlated with hypoxia. The objective of this study was to investigate the role of Jak2/STAT5b in the regulation of Hsp90 $\alpha$ expression so that Hsp90 $\alpha$ targeting can be achieved indirectly by modulating the Jak2/STAT5b pathway. We examined the role of the Jak2/STAT5b pathway in
\end{abstract}

Correspondence to: Dr Young Mok Yang, Department of Pathology, School of Medicine, and Institute of Biomedical Science and Technology, Konkuk University, Seoul 143-701, Republic of Korea E-mail: ymyang@kku.ac.kr

*Contributed equally

Abbreviations: STAT5b, signal transducer and activator of transcription 5b; Hsp $90 \alpha$, heat shock protein $90 \alpha$; GAS, interferon- $\gamma$ activation sites; HIF- $1 \alpha$, hypoxia inducible factor- $1 \alpha$; Jak2, janus kinase 2

Key words: heat shock protein $90 \alpha$, janus kinase $2 /$ signal transducer and activator of transcription $5 \mathrm{~b}$, hypoxia inducible factor-1 $\alpha$, hypoxia, cancer cells the expression of Hsp90 $\alpha$ under hypoxic conditions by immunoblotting, reporter gene assays, EMSA and RNA interference analysis. With the help of in vivo models, we also analyzed the expression of Hsp90 $\alpha$ in different parts of solid tumor tissues. We found a close association between hypoxic stress and Hsp90 $\alpha$ expression. We also determined that STAT5b regulates the expression of Hsp90 $\alpha$ during hypoxic stimulation. Under hypoxic conditions the expression of Hsp90 $\alpha$ and STAT5b were proportional. siRNA analysis and nucleotide analysis showed that the promoter of Hsp $90 \alpha$ has a STAT5b binding domain. Our work confirmed that STAT5b is one of the transcription factors that regulate $\mathrm{Hsp} 90 \alpha$. We, therefore, concluded that under hypoxic conditions, the Jak2/STAT5b pathway regulates Hsp90 $\alpha$ expression and it could serve as a promising target for the treatment of solid tumors.

\section{Introduction}

Chaperons are a class of proteins involved in the proper folding, intracellular localization, maturation, proteolytic turnover and prevention of improper association of several proteins (1). In most cell types, the heat shock protein (Hsp) or stress protein levels increase dramatically after exposure to environmental stress. These Hsp can act as molecular chaperons (2). Hsp is expressed normally at a basal level, is involved in protein folding and protects against misfolding and aggregation. Under various stress conditions, the expression level of Hsp increases and enhances cell survival (2). In this sense, chaperones seem to serve at the phenotypic level as biochemical buffers against numerous genetic lesions that are characteristic of most human cancers. These genetic lesions cause almost all cancer cells to acquire different characteristics such as growth signaling selfsufficiency, insensitivity to anti-growth signaling and apoptosis, 
persistent angiogenesis and the ablity to invade, metastasize and continually replicate $(2,3)$. Hsp90 plays a primitive role in all of these processes. Two major cytoplasmic isoforms of Hsp90, Hsp90 $\alpha$ (inducible) and Hsp90 $\beta$ (constitutive), have been identified in mammalian cells including those of humans (4).

STAT proteins have pivotal role in proliferation, differentiation and apoptosis. These proteins may affect proliferation by regulating the expression of immediate-early genes, such as $c-m y c$ and $c$-fos, as well as those of cell cycle regulators such as cyclins (5). After ligands bind to the cognate receptors on the cell surface, STAT molecules are recruited to the cytoplasm and activated by the phosphorylation of specific tyrosine residues in the C-terminus. This activation can be mediated by the Tyr kinase activity of growth factor receptors or by cytoplasmic non-receptor kinases such as Src, Jak and Abl. Most human cancers are associated with anomalous activation of certain STAT proteins, predominantly STAT3 and STAT5 (5-8). As to STAT5, there are two isotypes, STAT5a and STAT5b. In the Hsp90a and Jak/STAT pathway, we paid attention to STAT5b, but not STAT5a. Because, in the results of the previous experiment, it was the fact that the correlation of the STAT5b with hypoxia is higher than STAT5a (9-12).

The Jak/STAT pathways are activated by various factors and cytokines leading to the activation of Jak tyrosine kinase followed by tyrosine phosphorylation of the receptors. These receptors then activate STAT5 transcription factors. Phosphorylated STATs then dimerize and translocate to the nucleus. Once inside the nucleus, these factors activate target genes by binding to promoter STAT response elements and induce the tumor physiological function. Jak2 mediates cell growth regulation through two STATs with a 95\% homology: STAT5a and STAT5b (13). Activated STAT molecules regulate gene transcription through the interaction with IFN- $\gamma$ activation site (GAS) or DNA binding domains (14). We have previously reported that the Jak2/STAT5b pathway regulates the transactivation of cyclin D1 and IGF-I signal pathways in solid tumor cells, especially under hypoxic conditions $(9-12,15,16)$, thus making STAT5b a promising target for blocking tumor growth.

Hypoxic stress is a common phenomenon observed in all solid tumors that inhibits cell survival. Cells respond to this stress by expressing a group of proteins known as hypoxia inducible factors (HIFs). HIF-1 $\alpha$ is a nuclear transcription factor involved in the transactivation of a number of target genes. This factor induces angiogenesis as an adaptation to hypoxia by triggering the expression of its target genes (17). Hsp90 interacts with HIF-1 $\alpha$ (18), induces the necessary conformational changes to HIF and recruits different cofactors (19). Recently, we also reported that STAT3 modulates VEGF through HIF-1 $\alpha$ (20). HIF-1 $\alpha$ is associated with VEGF expression and metastasis of different cancers.

Targeted cancer therapy is an approach for selectively acting upon different key regulators of cancer progression and metastasis, thereby making it possible to manage these deadly diseases. Different small molecule drugs are under trial for targeting Hsp90 in various cancers. Targeting Hsp90 will provide additional advantages since this can disrupt the activity of numerous receptors and transcription factors involved in oncogenesis. In the present study, we explored the mechanical role of STAT5b in the induction of Hsp90 $\alpha$ during hypoxia.

\section{Materials and methods}

Ethics statement. All procedures for animal experiment were approved by the Committee on the Use and Care on Animals (Institutional Animal Care and Use Committee, Seoul, Korea) and performed in accordance with the institution guidelines.

Antibodies and reagents. Penicillin-streptomycin solution and fetal bovine serum (FBS) were purchased from Hyclone (Logan, UT). Dulbecco's modified Eagle's medium (DMEM), RPMI-1640 medium and trypsin-EDTA were purchased from Gibco-BRL (Grand Island, NY). Anti-actin antibody and phosphate buffered saline (PBS) (pH 7.4) were obtained from Sigma Chemical Co. (St. Louis, MO). Hsp90 $\alpha$ polyclonal antibodies was purchased from Streesgen (Ann Arbor, MI). Anti-STAT5b, HIF-1 $\alpha$ antibodies and secondary antibody (goat anti-mouse and goat anti-rabbit IgG-horseradish peroxidase) were obtained from Santa Cruz Biotechnology (Santa Cruz, CA). Chemiluminescence (ECL) detection kit was purchased from Amersham Pharmacia Biotech (Piscataway, NJ). Restore ${ }^{\mathrm{TM}}$ western blot stripping buffer was purchased from Pierce (Rockford, IL). Qiaprep spin miniprep kit was purchased from Qiagen (Hilden, Germany). Luciferase assay (LUC assay) cell lysis kit and substrate were purchased from Promega Corp. (Madison, WI). The electrophoretic mobility shift assay (EMSA) kit and oligonucleotide probes (STAT5) were purchased from Pamomics (Fremont, CA). FuGene6 transfection reagent was from Roche (Basel, Switzerland), Paraformaldehyde and mounting solution in immunohistochemistry (IHC) were purchased from Dae Jung Chemicals \& Metals Co. (Shiheungcity, Korea) and Life Science (Mukilteo, WA). Triton X-100 was obtained from Sigma Chemical Co.

Cell culture and transient transfection. MDA-MB 231, human breast cancer cells and COS-7, monkey kidney cells were grown to confluency in DMEM containing 10\% FBS, $2 \mathrm{mM}$ glutamine and $100 \mathrm{U} / \mathrm{ml}$ penicillin and streptomycin at $37^{\circ} \mathrm{C}$ in 5\% $\mathrm{CO}_{2}$. MCF-7 and SK-BR3, human breast cancer cells, HepG2, human liver cancer cells and 253J-BV and T24 human bladder cancer cells were grown to confluency in RPMI-1640 containing 10\% FBS, $2 \mathrm{mM}$ glutamine and $100 \mathrm{U} / \mathrm{ml}$ penicillin and streptomycin at $37^{\circ} \mathrm{C}$ in $5 \% \mathrm{CO}_{2}$. At the initiation of each experiment, the cells were resuspended in the medium at a density of $2.5 \times 10^{5}$ cells $/ \mathrm{ml}$. For hypoxic conditions, the cells were placed in airtight chambers (NuAire, Plymouth, MN) that were flushed with a $5 \%$ carbon dioxide $/ 95 \%$ nitrogen mixture until the oxygen concentration was $<2 \%$. For transfection, MDA-MB 231 or COS-7 cells into 35-mm culture dishes were transfected with RNA or DNA constructs (RNA interference $20 \mathrm{pM} / \mathrm{ml}$, reporter gene assay $1.0 \mu \mathrm{g}$ ) using the FuGene6 according to the manufacturer's recommendation.

Total cell lysis and immunoblots. MDA-MB 231 cells and other cells, respectively, were induced under normoxic and hypoxic condition for determined times. Cells were lysed ice on with radioimmunoprecipitation assay (RIPA) lysis buffer, containing protease and phosphatase inhibitors. Cells were disrupted by aspiration through a 23 -gauge needle and centrifuged at $15,000 \mathrm{rpm}$ for $10 \mathrm{~min}$ at $4^{\circ} \mathrm{C}$ to remove cellular debris. All processes for extracting total protein were at $4^{\circ} \mathrm{C}$. The extracted 
protein concentrations were measured using the Bradford method. An equivalent amount of protein extract from each sample was electrophoresed by $10 \%$ SDS-PAGE and transferred to nitrocellulose. Membranes were blocked for $1 \mathrm{~h}$ with $5 \%$ non-fat milk or $5 \%$ bovine serum albumins (BSA) in T-TBS buffer (20 mM Tris- $\mathrm{HCl}$ pH 7.6, $137 \mathrm{mM} \mathrm{NaCl}, 0.1 \mathrm{X}$ Tween20) and incubated overnight at $4^{\circ} \mathrm{C}$ with primary antibodies. The membranes were probed with primary antibodies followed by HRP conjugated secondary antibodies. Antibody detection was done by using enhanced chemiluminescence (ECL) plus detection kit.

Tumorigenicity. MDA-MB 231 tumor xenografts were established by subcutaneously inoculating $1 \times 10^{7}$ cells into the right flank of 5-week-old BALB/c nude mice (Orient Bio, Seongnam-Si, Korea). Animals were sacrificed when the diameter of tumors reached $2 \mathrm{~cm}$ or after 30 days. In our experiments, no mouse was observed dead due to tumor load. All available breast cancer specimens collected from in human breast cancer xenograft mice were reviewed and included in the study. Mice were euthanized and the tumors were removed. The tumors were fixed with $4 \%$ paraformaldehyde followed by paraffin-embedding and sectioning $(5 \mu \mathrm{m})$.

Immunohistochemistry. Formalin-fixed paraffin-embedded xenografts were sliced into $5 \mu \mathrm{m}$ thick tissue sections. The tissue sections were deparaffinized with xylene (100\%), rehydrated with decreasing concentrations $(100 \%, 90 \%, 80 \%$ and $70 \%)$ of ethyl alcohol, permeabilized with Triton X-100 (0.1\%) and blocked with NGS (10\% normal goat serum in PBS), then incubated in a closed humid chamber with the Hsp90 $\alpha$, STAT5b and HIF-1 $\alpha$ antibody followed by secondary antibody, Alexa Fluor 488 (rabbit) and Alexa Fluor 594 (mouse) (Invitrogen, Carlsbad, CA). For nuclear staining, tissue sections were incubated with DAPI for $1 \mathrm{~min}$ and rinsed with PBS. The slides were then observed under a fluorescent microscope.

Co-transfection and luciferase reporter assay. COS-7 cells were transiently co-transfected with Hsp90 $\alpha$ (kindly provided by $\mathrm{Dr}$ Carrie Shemanko, Calgary University, Calgary, AB, Canada) in 35-mm culture dishes and STAT5b (pMX/STAT5b; kindly provided by Dr Koichi Ikuta, Kyoto University, Japan) recombinant DNA constructs $(1 \mu \mathrm{g})$ using the FuGene6 according to the manufacturer's recommendation. Transfected cells were washed twice with ice-cold PBS and $120 \mu \mathrm{l}$ of cell culture lysis buffer reagent was added to each dish. Lysates were then used directly to measure luciferase activity. For luciferase assays, $25 \mu \mathrm{l}$ of cell lysates was mixed with $25 \mu \mathrm{l}$ of luciferase assay substrate. Luciferase activity of each sample was determined by measuring Veritas ${ }^{\mathrm{TM}}$ Microplate Luminometer (Turner Biosystems Inc., Sunnyvale, CA) then the mixture of lysates and substrate was loaded in Lumitrac ${ }^{\mathrm{TM}}$ 96-well microplates (Greiner Bio-One, Frickenhausen, Germany). The experiments were performed in triplicate and similar results were obtained from at least three independent experiments.

Electrophoretic mobility shift assay (EMSA). STAT5b DNA binding activity was detected using the electrophoretic mobility shift assay (EMSA), in which a labeled double-stranded DNA sequence was used as DNA probe to bind with active STAT5b protein in nuclear extracts. Nuclear protein extracts were prepared with the Nuclear Extract Kit (Panomics, AY2002). EMSA experiment is performed by incubating a biotin-labeled transcription factor (TF-STAT5) probe with induced and not induced nuclear extracts. EMSA was performed under the following conditions: MDA-MB 231 cells were grown to $90 \%$ confluence. Reactions were resolved on a nondenaturing $4 \%$ to $20 \%$ PAGE gel (Bio-Rad, Korea) and mobility shifts were detected by chemiluminescence. The proteins gel is transferred to a nylon membrane and detected using strepatvidin-HRP and a chemiluminescent substrate. The shifted bands corresponding to the protein/DNA complexes visualized were related to the unbound dsDNA.

RNA interference. MDA-MB 231 cells were transfected with ON-TARGETplus SMARTpool siRNA targeting human STAT5b and ON-TARGETplus Non-targeting siRNA (ON-TARGETplus SMARTpool siRNA, ON-TARGETplus Control) purchased from Dharmacon (Lafayette, CO). Transfections were performed by using the FuGene6 according to the manufacturer's recommendation. MDA-MB 231 cells were plated at $5 \times 10^{6}$ and transfected with STAT5b siRNA $(20 \mathrm{pM} / \mathrm{ml})$ or control siRNA $(20 \mathrm{pM} / \mathrm{ml})$ using the FuGene6 in a $35-\mathrm{mm}$ culture dish on the serum-free media. Forty-eight hours after transfection, cells were placed in hypoxia chamber for inducing hypoxic condition.

Statistical analysis. The results of the experiments are expressed as mean \pm SEM. Statistical analysis was done with Student's t-test or ANOVA test of the SAS program. These were compared by one-way analysis of variance followed by Duncan's multiple range test.

\section{Results}

Hsp90 a expression is increased more in aggressive cancer cell lines under hypoxic conditions than in non-aggressive cancer cell lines. The role of hypoxia in the expression of Hsp90 $\alpha$ was evaluated using six different human cancer cell lines (aggressive cell lines: MDA-MB 231, SK-BR3, 253J-BV; non-aggressive cell lines: MCF-7, HepG2, T24). The expression patterns of Hsp90 $\alpha$ were determined in both aggressive (Fig. 1A) and non-aggressive (Fig. 1B) cancer cells. We found that hypoxia upregulated the expression of Hsp90 $\alpha$ regardless of cell type. In comparison, aggressive cancer cell lines expressed greater amounts of Hsp90a, showing that they are more sensitive to hypoxic stress than non-aggressive cancer cell lines.

Hypoxia upregulates Hsp $90 \alpha$ expression in vivo. The role of hypoxia in the upregulation of Hsp90 $\alpha$, STAT5b and HIF-1 $\alpha$ expression was analyzed in vivo. Breast tumor xenografts were established in Balb/c athymic nude mice and maintained for 30 days. After this, the xenografts were surgically removed and subjected to protein expression profiling. The tumor xenograft was split into small pieces and marked as inner and outer areas according to the site that they were obtained. The samples were assayed separately as they were believed to have different grades of angiogenesis. The tissue samples were subjected to immunohistochemistry with primary antibodies specific for STAT5b, Hsp90 $\alpha$ and HIF-1 $\alpha$. The samples were also stained 
A
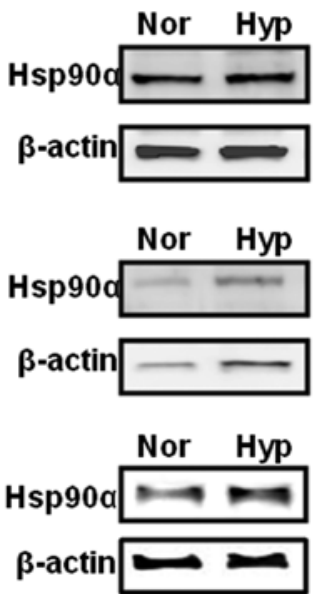

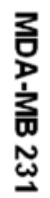

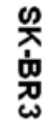

N
B
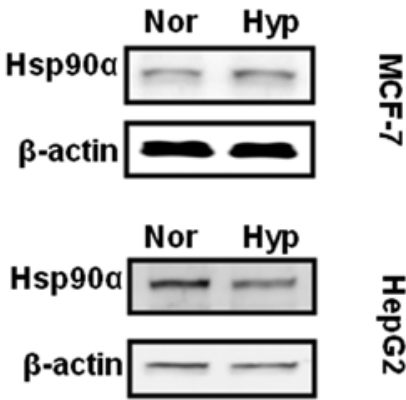

กํํำ

$\underset{+}{\stackrel{5}{+}}$

Figure 1. Hsp90 $\alpha$ expression is increased more in aggressive cancer cell lines under hypoxic conditions than in non-aggressive cancer cell lines. (A) The expression of Hsp90 $\alpha$ in aggressive cancer cells under normoxic and hypoxic condition in $24 \mathrm{~h}$ (human breast cancer cells: MDA-MB 231, SK-BR-3; human bladder cancer cells: 253J-BV). (B) The expression of Hsp90 $\alpha$ in non-aggressive cancer cells normoxic and hypoxic condition in $24 \mathrm{~h}$ (human breast cancer cells: MCF7; human liver cancer cells: HepG2; human bladder cancer cells: T24). The whole cell extracts were separated by $10 \%$ SDS-PAGE and immunoblots were performed as described in experimental procedures. $\beta$-actin was used as a control for protein loading. Data are one representive of three independent experiments.

A
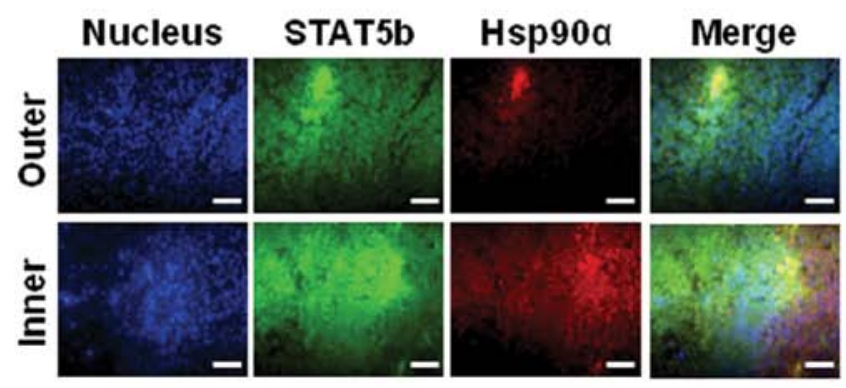

C

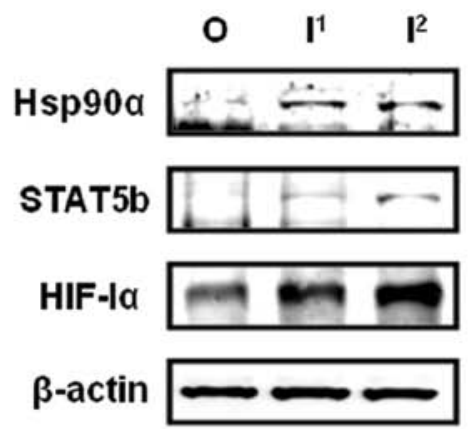

D

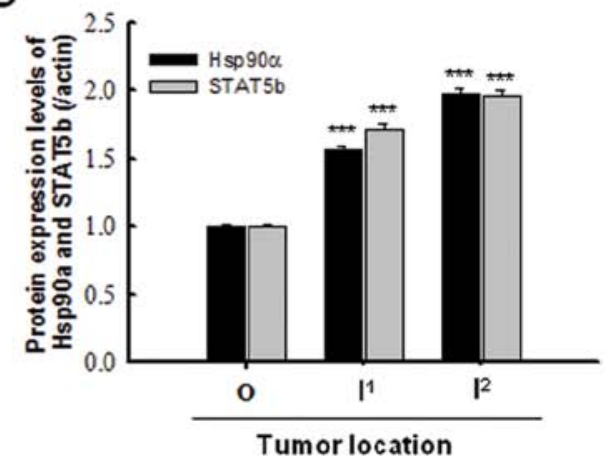

Figure 2. Hypoxia upregulates Hsp90 $\alpha$ expression in vivo. The xenografts were sliced to $5 \mu \mathrm{m}$ thickness and treated with primary antibodies specific for STAT5b, Hsp $90 \alpha$ and HIF-1 $\alpha$. Detection was done using secondary antibody, Alexa Fluor 488 (rabbit) and Alexa Fluor 594 (mouse). Studies confirmed the increase in expression of STAT5b, Hsp90 $\alpha$ and HIF-1 $\alpha$ with no much alteration in the nucleus level. (A) IHC specific for STAT5b/Hsp90 $\alpha$ and (B) IHC specific for STAT5/ HIF-1 $\alpha$. (C) Immunoblotting of tissue extracts specific for Hsp90 $\alpha$ and HIF-1 $\alpha$. (D) The relative expression levels of proteins (density/actin). Data represent the mean of at least three separate experiments, mean \pm SEM. ${ }^{* * *}$ p $<0.001$ indicates a statistically significant decrease by ANOVA test. Scale bar, $50 \mu \mathrm{m}$. O, outer part of breast cancer xenografts; $\mathrm{I}^{1}$ and $\mathrm{I}^{2}$, separated inner part of breast cancer xenografts.

with nuclear-specific DAPI. Our results showed an increase in the expression of STAT5b, Hsp90 $\alpha$ and HIF- $1 \alpha$ in the inner part of the tissue compared to the outer part (Fig. 2A and B).
Immunoblotting of these samples showed that the inner hypoxic counterpart $\left(\mathrm{I}^{1}\right.$ and $\left.\mathrm{I}^{2}\right)$ of the tissue samples had higher expression levels of Hsp90 $\alpha$, STAT5b and HIF-1 $\alpha$ than the 
A
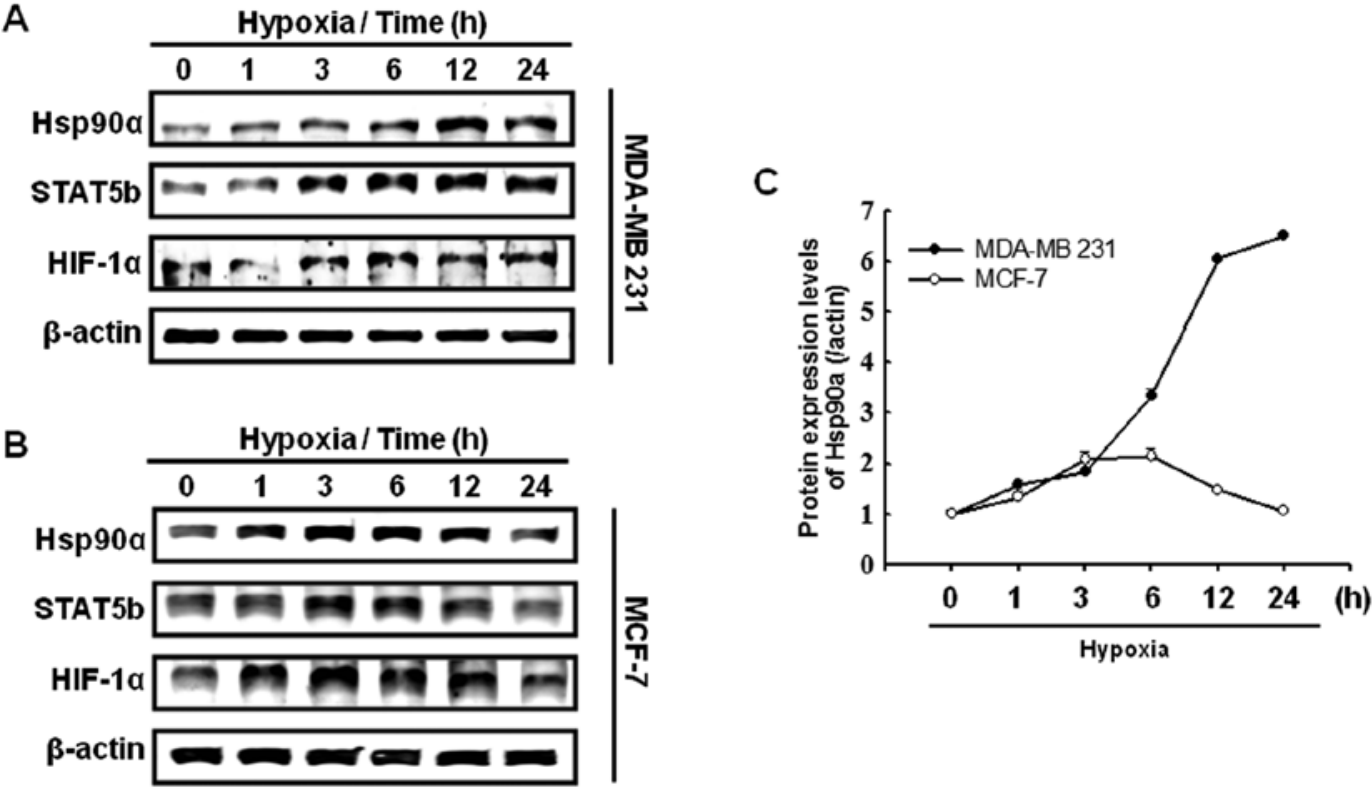

Figure 3. Hypoxia upregulates the expression of Hsp90 $\alpha$, STAT5b and HIF-1 $\alpha$ in a time dependent manner. (A and B) The expression of Hsp90 $\alpha$, STAT5b and HIF-1 $\alpha$ under hypoxic condition of MDA-MB 231 and MCF-7 cells in a time course manner. Both cells were treated under normoxic (20\% $\mathrm{O}_{2}$ ) and hypoxic conditions (less $2 \% \mathrm{O}_{2}$ ) for 0 (Nor), 1, 3, 6, 12 and $24 \mathrm{~h}$. Immunoblots were performed as described above. (C) The relative expression levels of proteins (density/actin) in time course manner. Data are one representative of three independent experiments. $0 \mathrm{~h}$, normoxia.

peripheral regions (Fig. 2C). The relative expression also showed a statistically significant reduction in the expression of STAT5b and Hsp90 $\alpha$ in the outer part of the xenograft compared to the interior (Fig. 2D, ${ }^{* * *} \mathrm{p}>0.001$ ). This difference in expression may be due to the lesser degree of angiogenesis in the interior of the tumors. The comparatively peripheral regions of the tissue had well-established capillaries that supplied oxygen and nutrients. In the interior of the tumors, the tissues were oxygen-starved due to the small number of capillaries. This might have induced hypoxic stress in the xenograft that led to the upregulation of Hsp90 $\alpha$ and related molecular expression.

Hypoxia upregulates the expression of Hsp $90 \alpha$, STAT5b and $H I F-1 \alpha$ in a time-dependent manner. Increased expression of Hsp90 $\alpha$ under hypoxic condition has been reported in breast cancer (21-23). We found that hypoxia led to the upregulated expression of Hsp90 $\alpha$, STAT5b and HIF- $1 \alpha$ in MDA-MB 231 cells (Fig. 3A). To confirm that hypoxia has a consistent effect on the expression of survival and oncogenic proteins, we evaluated the impact of hypoxia in the non-aggressive breast cancer cell line MCF-7 (Fig. 3B). The cell lines were kept under hypoxic condition, defined as $2 \% \mathrm{O}_{2}$ for $0,1,3,6,12$ and $24 \mathrm{~h}$. We observed a time-dependent upregulation of protein expression irrespective of cell line characteristics (Fig. 3). We also determined that the level of Hsp90 $\alpha$ expression increased steadily until $12 \mathrm{~h}$ in aggressive cancer cells whereas Hsp90 $\alpha$ levels increased until $6 \mathrm{~h}$ and then decreased in non-aggressive cancer cells (Fig. 3C). These results indicate that the expression of Hsp90 $\alpha$ increases together with that of STAT5b. This may occur because STAT5b binds to the GAS promoter region of Hsp90 $\alpha$ and thereby promotes Hsp90 $\alpha$ expression.

Hypoxia increases STAT5b/Hsp $90 \alpha$ promoter activity. The effect of hypoxia on the post-transcriptional activation of
Hsp90 $\alpha$ was examined using a human Hsp90 $\alpha$ promoterluciferase construct (kindly provided by Dr Carrie Shemanko; Fig. 4A). For transient co-transfection, a genomic DNA fragment was sub-cloned upstream of a promoterless firefly luciferase reporter in the pGL2 vector. The promoter activities of these fragments have been previously described (24). COS-7 cells were transiently co-transfected with the Hsp90 $\alpha$ and STAT5b constructs. They were incubated under normoxic or hypoxic conditions for $12 \mathrm{~h}$. As shown in Fig. 4B, the relative luciferase activity of STAT5b/Hsp90 $\alpha$ cells under hypoxic conditions increased compared to those grown under normoxic conditions. In particular, the relative luciferase activity (the reporter gene expression) of the STAT5b/Hsp90 $\alpha$ cells slightly increased in the presence of hypoxia compared to STAT5b/Hsp90 $\alpha$ cells grown in normoxia $\left({ }^{* * *} \mathrm{p}<0.001\right)$. These results suggest that the STAT5b protein may be a significant regulator of Hsp90a expression under hypoxic conditions (Fig. 4B).

Hypoxia increases the binding of STAT5 to the consensus sequence in the Hsp $90 \alpha$ promoter. The Jak2/STAT5b signaling pathway contributes to oncogenic transformation. Activated STATs form dimers, translocate into the nucleus, bind to specific response elements in the promoters of target genes and activate those genes (15). The DNA binding activity of aggressive cancer cells corresponded to a supershift in the EMSA assay (Fig. 5B, right) corresponding to the interaction of STAT $5 b$ with the GAS element (GAAaagTTC) of Hsp90 $\alpha$ (Fig. 5A). We found that the expression as well as the binding activity of STAT5b was increased by hypoxic stress (Fig. 5B, left). The nuclear extracts of aggressive cancer cells also showed increased expression of STAT5b and Hsp90 $\alpha$ under hypoxic conditions (Fig. 5C).

Expression of Hsp $90 \alpha$ is significantly reduced by transfection of cancer cells with STAT5b siRNA under hypoxic conditions. 
A

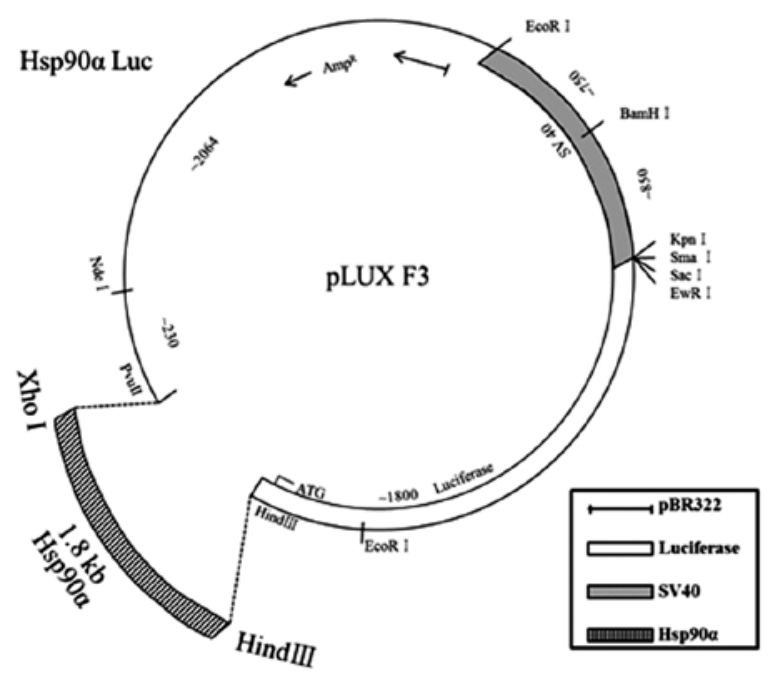

B

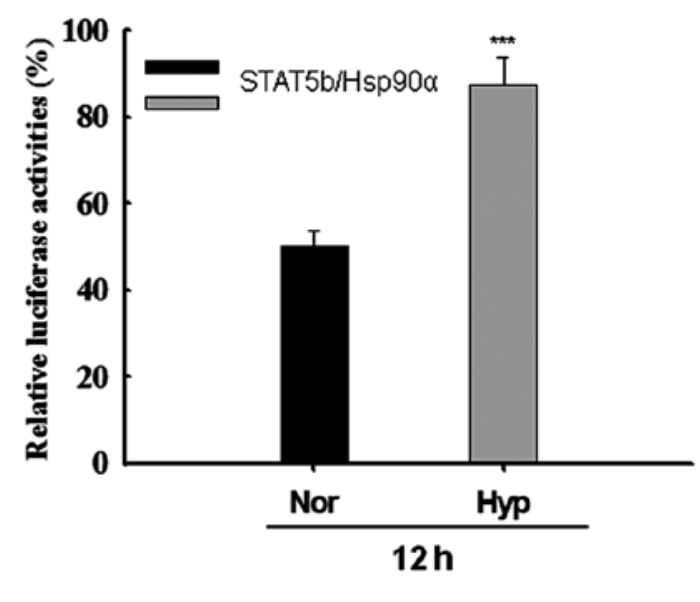

Figure 4. Hypoxia increases STAT5b/Hsp90 $\alpha$ promoter activity. (A) Map of the Hsp90 $\alpha$-luciferase recombinant genomic DNA construct. The recombinant luciferase DNA construct was made including the human Hsp90 $\alpha$ sequence for reporter gene assay. The recombination vector contained the human Hsp90 $\alpha$ sequence which has $1.8 \mathrm{~kb}$ size after restricted and ligated the pLUX F3 vector with the restriction and ligation enzyme. (B) Hsp90 $\alpha /$ STAT5b promoter activities compared with $12 \mathrm{~h}$ under normoxic or hypoxic conditions. Hsp90 $\alpha /$ STAT5b promoter activity increased under hypoxic condition. COS-7 cells were transiently co-transfected with an Hsp90 $\alpha$ and STATb luciferase reporter gene and then cultured in serum-free media, exposed to normoxic condition or hypoxic condition for $12 \mathrm{~h}$. Cell lysates were assayed for luciferase activity using a luminometer. Data represent the mean of at least three separate experiments, mean \pm SEM. ${ }^{* * * *}$ p $<0.001$ indicates a statistically significant increase of promoter activity by Student's t-test between normoxic condition and hypoxic condition.

A

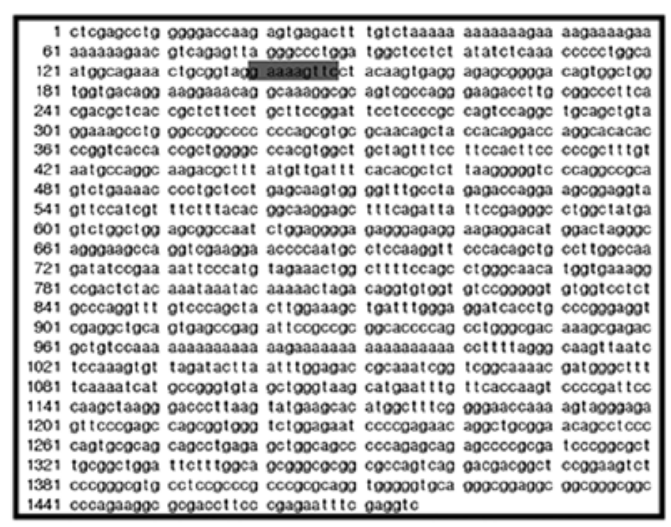

gaaagittc: STAT5b binding sequences

C

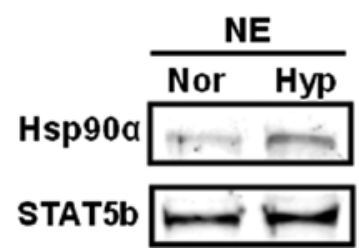

B

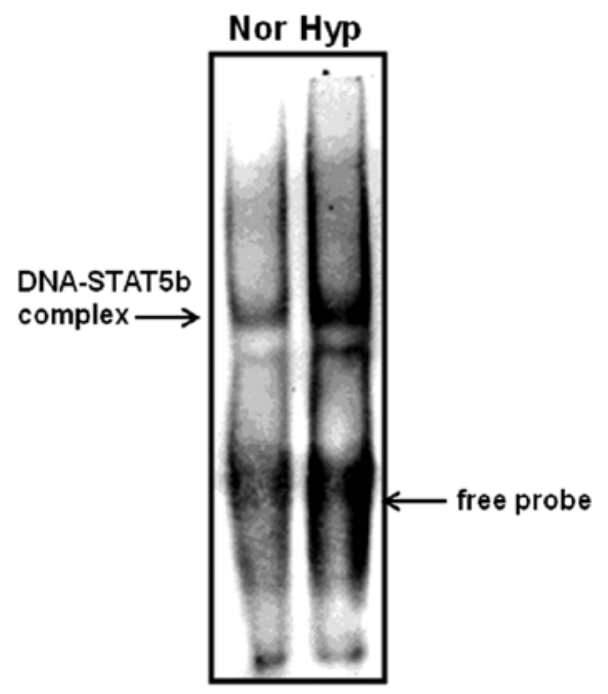

Nor Hyp+Ab

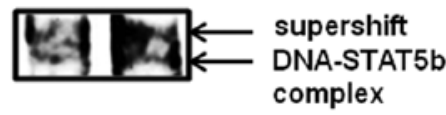

Figure 5. Hypoxia increases the binding of STAT5 to the consensus sequence in the Hsp90 $\alpha$ promoter. (A) Sequence of the human $H s p 90 \alpha$ gene promoter (NCBI GenBank U25822gi/793941/gb/U25822.1/HSU25822, TESS). GAS element of $H s p 90 \alpha$ gene is highlighted, and is present at nucleotide sequence 140-148. (B) STAT5 binding to the $H s p 90 \alpha$ gene promoter and more increased activity of the binding of STAT5b to the Hsp $90 \alpha$-binding site under hypoxic condition than normoxic condition in $12 \mathrm{~h}$ as shown by EMSA. (C) nuclear protein extracts (NE) were separated and blotted onto nitrocellulose membrane showing decrease in the level of Hsp90 $\alpha$ and STAT5b under normoxic and hypoxic condition in $12 \mathrm{~h}$. Data are one representative of three independent experiments.

The role of STAT5b in Hsp90 $\alpha$ expression was studied by siRNA analysis. The STAT5b gene was silenced by transfecting MDA-MB 231 cells with human STAT5b siRNA for $12 \mathrm{~h}$. This led to the efficient and specific inhibition of Hsp90 $\alpha$ expression (Fig. 6A). Knock down of STAT5b did not affect the expression of $\beta$-actin. The relative expression of STAT5b and Hsp90 $\alpha$ with respect to hypoxia was significantly reduced according to ANOVA analysis (Fig. 6B, ${ }^{* * *} \mathrm{p}<0.001$ ). These 
A

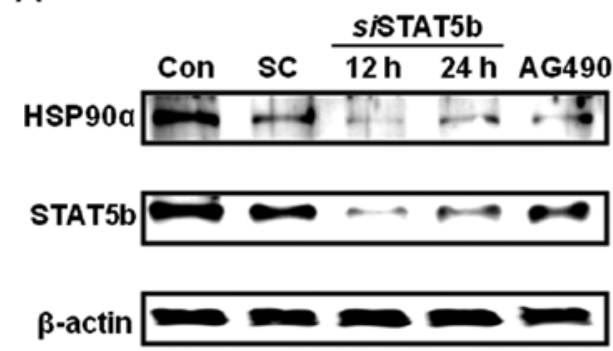

B

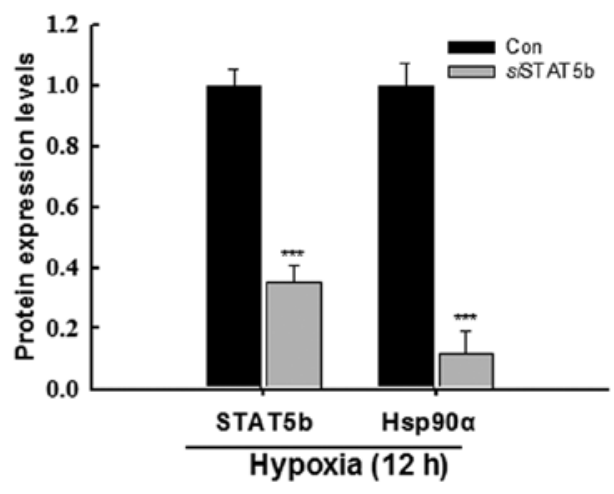

Figure 6. Expression of Hsp90 $\alpha$ is significantly reduced by transfection of cancer cells with STAT5b siRNA under hypoxic conditions. (A) Treatment with STAT5b siRNA reduced expressions of target Hsp90 $\alpha$ under normoxic or hypoxic conditions in MDA-MD 231 cells. MDA-MD 231 cells were treated with scramble (non-targeting siRNA) or siSTAT5b (human STAT5b siRNA) under normoxic and hypoxic conditions. Cells were harvested after $12 \mathrm{~h}$ for assay of Hsp90 $\alpha$, STAT5b and $\beta$-actin by immunoblotting. (B) Patterns of the Hsp90 $\alpha$ and STAT5b expression after knock-down STAT5b under normoxic and hypoxic conditions. ${ }^{* * * *} \mathrm{p}<0.001$ indicates statistically significant decreases of STAT5b and Hsp90 $\alpha$ protein expression after siRNA STAT5b by ANOVA test. The error bars represent the standard deviation among three experiments. Con, control; SC, scramble; siSTAT5b, STAT5b siRNA.

data showed that, hypoxia induced the dimerization and binding of STAT5b to the GAS element of Hsp90 $\alpha$.

\section{Discussion}

STAT5b is a transcription factor essential for the proliferation and survival of many solid tumors (25). Overexpression of STAT5b is found in hematopoietic malignancies and solid tumors (25). In our previous study, we confirmed that hypoxia stimulates phosphorylation and activation of Jak2 and STAT5b in breast cancer cells $(9,12,15)$. The activation of and phosphorylation of STAT5b is mediated by several kinases that are overexpressed in breast cancer. Jak2 is a kinase involved in the activation and phosphorylation of STAT. Induction of STAT under hypoxic conditions is mediated mainly through HIF-1 $\alpha$.

In this present study, we observed the overexpression of HIF-1 $\alpha$ in response to hypoxia. Overexpression of HIF-1 $\alpha$ is associated with angiogenesis and tumorigenesis via the transcriptional activation of VEGF (26). Erythropoietin (Epo) is a major target of HIF-1 $\alpha$, which stimulates Jak2 phosphorylation of I- $\kappa \mathrm{B}$. This will in turn release NF- $\kappa \mathrm{B}(27)$ which is then translocated into the nucleus and regulates the Hsp90 $\alpha$ promoter (4).

In order to determine whether the effect of hypoxia was cell line-specific or not, we conducted protein expression analysis in different aggressive and non-aggressive cancer cell lines. We observed a time-dependent increase in STAT5b expression. We also found that the expression of Hsp90 $\alpha$ was comparatively higher in aggressive cancers cells than that in non-aggressive cancers cells (Fig. 1). We observed the same effects of hypoxia in solid tumors. In our experimental animal models, we found an inverse relationship of hypoxia and Hsp90 $\alpha$ expression. When oxygen availability in the inner area of the tumor was reduced, the expression levels of Hsp90 $\alpha$, STAT5b and HIF-1 $\alpha$ increased (Fig. 2).

We previously reported that hypoxia activates STAT5 and enhances its binding to the GAS sequence in mammary epithelial cells (15). In the present study, we found that hypoxia upregulated the expression of STAT5b regardless of aggressive cell type in breast cancer (Fig. 3). To identify the effects of elevated STAT5b on Hsp90 $\alpha$ gene expression in the presence of hypoxic stress, promoter activities of Hsp90 $\alpha$ gene were determined. Our luciferase assay showed that STAT5b has a statistically significant effect on the modulation of Hsp90 $\alpha$ expression under hypoxic conditions (Fig. 4B, ${ }^{* * *} \mathrm{p}<0.001$ ). This finding indicated that there is another pathway which regulates Hsp $90 \alpha$ expression apart from NF- $\mathrm{BB}$ signaling, the major mechanism of $\mathrm{Hsp} 90 \alpha$ regulation under hypoxic conditions.

When analyzing the DNA sequence of the human Hsp90 $\alpha$ gene promoter (NCBI GenBank U25822gi/793941/gb/ U25822.1/HSU25822, TESS), we identified a STAT5b binding motif. This GAS element was present between the sequences 140-148. We subsequently performed a DNA binding assay specific for STAT5b in the Hsp90 $\alpha$ gene promoter. The results of this assayed show a high level of interaction of STAT5b and the Hsp90 $\alpha$ promoter (Fig. 4B). STAT5b-specific RNA interference studies highlighted the importance of STAT5b interaction with GAS element for the regulation of Hsp90 $\alpha$ expression (Fig. 5A and B). These experiments confirmed the role of STAT5b in regulating the expression level of Hsp90 $\alpha$ under hypoxic conditions. The role of STAT1 in regulating the expression of Hsp90 $\alpha$ under heat stress has already been confirmed (28). This study, along with the results from the present study, indicates that members of STAT family are important target molecules for the regulation of stress-related factors.

Interaction of STAT5b with the promoter site of the Hsp90 $\alpha$ gene is the basic requirement for appropriate expression of Hsp90 $\alpha$ under hypoxic conditions. Therefore, regulating STAT5b expression and activation by Jak2 is promising for different therapeutic measures. Hsp90 $\alpha$ is a molecular chaperon associated with protein folding along with inhibition of protein misfolding and aggregation (1). Expression of Hsp90 $\alpha$ also associated with different stress factors and leads to tumor progression, invasion and metastasis (29). The association of Hsp $90 \alpha$ with VEGF through HIF- $1 \alpha$ is found in many solid tumors (2). VEGF is the important pro-angiogenenic factor expressed in all forms of malignancies and under different 
physiological condition leading to arteriogenesis (30). Hence, we suggest that targeting the Jak2/STAT5b pathway is the most suitable approach for breast cancer management. Therapies targeting STAT5b may effectively control breast cancer through modulating Hsp90 $\alpha$.

In conclusion, we have shown for the first time that STAT5b is a functional regulator of $\mathrm{Hsp} 90 \alpha$ under hypoxic conditions. The efficiency of STAT5b induction in response to hypoxic stress depends on the duration of the stress. Based on our findings, we propose that this represents an alternative pathway independent of NF- $\mathrm{kB}$ dependent activation for the regulation of Hsp90 $\alpha$ under hypoxic conditions.

\section{Acknowledgements}

This study was supported by a grant from the Next generation BioGreen 21 Program (no. PJ0081062011), Rural Development Administration, and partially supported by the Ministry of Commerce, Industry and Energy through the Bio-Food and Drug Research Center at Konkuk University Glocal Campus, Republic of Korea.

\section{References}

1. Whitesell L and Lindquist SL: HSP90 and the chaperoning of cancer. Nat Rev Cancer 5: 761-772, 2005.

2. Neckers L: Heat shock protein 90: the cancer chaperone. J Biosci 32: 517-530, 2007

3. Hanahan D and Weinberg RA: The hallmarks of cancer. Cell 100: 57-70, 2000.

4. Ammirante M, Rosati A, Gentilella A, Festa M, Petrella A, Marzullo L, Pascale M, Belisario MA, Leone A and Turco MC: The activity of hsp90 alpha promoter is regulated by NF-kappa B transcription factors. Oncogene 27: 1175-1178, 2008.

5. Bowman T, Garcia R, Turkson J and Jove R: STATs in oncogenesis. Oncogene 19: 2474-2488, 2000.

6. Turkson J and Jove R: STAT proteins: novel molecular targets for cancer drug discovery. Oncogene 19: 6613-6626, 2000.

7. Levy DE and Darnell JE Jr: Stats: transcriptional control and biological impact. Nat Rev Mol Cell Biol 3: 651-662, 2002.

8. Yu H and Jove R: The STATs of cancer - new molecular targets come of age. Nat Rev Cancer 7: 41-51, 2004.

9. Joung YH, Lim EJ, Lee MY, Park JH, Ye SK, Park EU, Kim SY, Zhang Z, Lee KJ, Park DK, Park T, Moon WK and Yang YM: Hypoxia activates the cyclin D1 promoter via the Jak2/STAT5b pathway in breast cancer cells. Exp Mol Med 37: 353-364, 2005.

10. Joung YH, Lee MY, Lim EJ, Kim MS, Hwang TS, Kim SY, Ye SK, Lee JD, Park T, Woo YS, Chung IM and Yang YM Hypoxia activates the IGF-1 expression through STAT5b in human HepG2 cells. Biochem Biophys Res Commun 358: 733-738, 2007.

11. Joung YH, Lim EJ, Kim MS, Lim SD, Yoon SY, Lim YC, Yoo YB, Ye SK, Park T, Chung IM, Bae KY and Yang YM: Enhancement of hypoxia-induced apoptosis of human breast cancer cells via STAT5b by momilactone B. Int J Oncol 33: 477-484, 2008.

12. Lee MY, Joung YH, Lim EJ, Park JH, Ye SK, Park T, Zhang Z, Park DK, Lee KJ and Yang YM: Phosphorylation and activation of STAT proteins by hypoxia in breast cancer cells. Breast 15: 187-195, 2006.

13. Schwaller J, Parganas E, Wang D, Cain D, Aster JC, Williams IR, Lee CK, Gerthner R, Kitamura T, Frantsve J, Anastasiadou E, Loh ML, Levy DE, Ihle JN and Gilliland DG: Stat5 is essential for the myelo- and lymphoproliferative disease induced by TEL/JAK2. Mol Cell 6: 693-704, 2000.
14. Arnould C, Philippe C, Bourdon V, Gr goire MJ, Berger R and Jonveaux P: The signal transducer and activator of transcription STAT5b gene is a new partner of retinoic acid receptor alpha in acute promyelocytic-like leukaemia. Hum Mol Genet 8: 1741$1749,1999$.

15. Joung YH, Park JH, Park T, Lee CS, Kim OH, Ye SK, Yang UM, Lee KJ and Yang YM: Hypoxia activates signal transducers and activators of transcription 5 (STAT5) and increases its binding activity to the GAS element in mammary epithelial cells. Exp Mol Med 35: 350-357, 2003.

16. Lim EJ, Joung YH, Jung SM, Park SH, Park JH, Kim SY, Hwang TS, Hong DY, Chung SC, Ye SK, Moon ES, Park EU, Park T, Chung IM and Yang YM: Hemin inhibits cyclin D1 and IGF-1 expression via STAT5b under hypoxia in ER $\alpha$-negative MDA-MB 231 breast cancer cells. Int J Oncol 36: 1243-1251, 2010.

17. Patiar S and Harris AL: Role of hypoxia-inducible factor-1alpha as a cancer therapy target. Endocr Relat Cancer 13: S61-S75, 2006.

18. Gradin K, McGuire J, Wenger RH, Kvietikova I, Fhitelaw ML, Toftgård R, Tora L, Gassmann M and Poellinger L: Functional interference between hypoxia and dioxin signal transduction pathways: competition for recruitment of the Arnt transcription factor. Mol Cell Biol 16: 5221-5231, 1996.

19. Hur E, Kim HH, Choi SM, Kim JH, Yim S, Kwon HJ, Choi Y, Kim DK, Lee MO and Park H: Reduction of hypoxia-induced transcription through the repression of hypoxia-inducible factor1alpha /Aryl hydrocarbon receptor nuclear translocator DNA binding by the $90-\mathrm{kDa}$ heat-shock protein inhibitor radicicol. Mol Pharmacol 62: 975-982, 2002.

20. Jung JE, Lee HG, Cho IH, Chung DH, Yoon SH, Yang YM, Lee JW, Choi S, Park JW, Ye SK and Chung MH: STAT3 is a potential modulator of HIF-1-mediated VEGF expression in human renal carcinoma cells. FASEB J 19: 1296-1298, 2005.

21. Pick E, Kluger Y, Giltnane JM, Moeder C, Camp RL, Rimm DL and Kluger HM: High HSP90 expression is associated with decreased survival in breast cancer. Cancer Res 67: 2932-2937, 2007.

22. Kim HL, Cassone M, Otvos L Jr and Vogiatzi P: HIF-1alpha and STAT3 client proteins interacting with the cancer chaperone Hsp90: therapeutic considerations. Cancer Biol Ther 7: 10-14, 2008.

23. Trisciuoglio D, Gabellini C, Desideri M, Ziparo E, Zupi G and Del Bufalo D: Bcl-2 regulates HIF-1alpha protein stabilization in hypoxic melanoma cells via the molecular chaperone HSP90. PLoS One 5: e11772, 2010.

24. Sarfstein R, Maor S, Reizner N, Abramovitch S and Werner H: Transcriptional regulation of the insulin-like growth factor-I receptor gene in breast cancer. Mol Cell Endocrinol 252: 241-246, 2006

25. Bernaciak TM, Zareno J, Parsons JT and Silva CM: A novel role for signal transducer and activator of transcription $5 \mathrm{~b}$ (STAT5b) in beta1-integrin-mediated human breast cancer cell migration. Breast Cancer Res 11: R52, 2009.

26. Jung H, Lee E, Kim JW and Lee D: Pathway level analysis by augmenting activities of transcription factor target genes. IET Syst Biol 3: 534-542, 2009.

27. Paul I, Lappin TR, Maxwell P and Graham AN: Pre-operative plasma erythropoietin concentration and survival following surgery for non-small cell lung cancer. Lung Cancer 51: 329-334, 2006

28. Chen XS, Zhang Y, Wang JS, Li XY, Cheng XK, Zhang Y, Wu NH and Shen YF: Diverse effects of Stat1 on the regulation of hsp90 alpha gene under heat shock. J Cell Biochem 102: 1059-1066, 2007.

29. Eustace BK, Sakurai T, Stewart JK, Yimlamai D, Unger C, Zehetmeier C, Lain B, Torella C, Henning SW, Beste G, Scroggins BT, Neckers L, Ilag LL and Jay DG: Functional proteomic screens reveal an essential extracellular role for hsp90 alpha in cancer cell invasiveness. Nat Cell Biol 6: 507-514, 2004.

30. Buschmann I and Schaper W: Arteriogenesis versus angiogenesis: Two mechanisms of vessel growth. News Physiol Sci 14: 121-125, 1999. 